\title{
Range cell migration correction analysis of one-step and two-step motion compensation for millimeter-wave airborne SAR imaging
}

\author{
Guanyong Wang ${ }^{1,2}$, Lei Zhang ${ }^{1 *}$, Jun $\mathrm{Li}^{2}$ and Qingrong $\mathrm{Hu}^{2}$
}

\begin{abstract}
Conventional two-step motion compensation (MOCO) method is widely adapted for airborne synthetic aperture radar (SAR) imaging due to its conciseness combining with the SAR focusing procedure. For two-step MOCO, rangeindependent compensation is processed before range cell migration correction (RCMC), and the range-dependent phase correction is implemented after RCMC. However, the accuracy of RCMC would be seriously decreased by the residual range-dependent phase, which is a fatal problem for high-resolution millimeter-wave (MMW) SAR imaging. In this paper, an extensive investigation on the RCMC accuracy is provided by establishing an accurate formula expression between the range cell migration error and the residual range-dependent phase error. One-step MOCObased SAR imaging algorithm is investigated by compensating the range-dependent motion error before RCMC, so the presence of range cell migration error would be significantly suppressed. What is more, a modified azimuth match filtering (AMF) function is given by precise topography and aperture-dependent motion compensation (PTA) method to overcome the residual azimuth-dependent phase error in the azimuth compression stage. Both simulated and real-measured MMW SAR data sets are used to validate the analysis for high-resolution airborne SAR imaging.
\end{abstract}

Keywords: Synthetic aperture radar (SAR), Motion compensation (MOCO), Millimeter-wave (MMW), One-step MOCO

\section{Introduction}

Motion compensation (MOCO) [1-4] is a crucial operating step for airborne synthetic aperture radar (SAR) [5-7] imaging because the non-ideal movement deviates the radar platform from the predetermined flight trajectory. More importantly, for high-resolution millimeter-wave (MMW) [8] SAR systems, imaging performance is more sensitive to the envelope and phase of motion errors, so a precise MOCO is essential with the availability of high-precision inertial navigation system (INS) measurement. An efficient two-step MOCO algorithm [4] is proposed by Moreira and Huang, combining with chirp scaling algorithm (CSA) $[9,10]$ for airborne SAR imaging. This method is divided into range-independent compensation step and range-dependent compensation step,

\footnotetext{
*Correspondence: leizhang@xidian.edu.cn

${ }^{1}$ National Lab of Radar Signal Processing and the Collaborative Innovation

Center of Information Sensing and Understanding, Xidian University, Xi'an

710071, People's Republic of China

Full list of author information is available at the end of the article
}

while the first step is processed to the range compressed data and the second step is processed after range cell migration correction (RCMC). The problem of conventional two-step $\mathrm{MOCO}$ processing is also obvious. The residual range-dependent motion error remained after the first step seriously decreases the accuracy of RCMC in two-dimensional wavenumber domain, which presents as a curving range cell migration (RCM) range profile in two-dimensional time domain, and destroys the performance of azimuth pulse compression. Reference [11] describes the problem above for Omega-k algorithm [12, 13]. In their work, a one-step MOCO method is proposed, but the detailed analysis of the RCMC error is not given. Besides, the original MOCO methods only take range-dependent motion error into account, and the residual azimuth-dependent motion error should also be considered, which is nonignorable for high-resolution airborne MMW SAR imaging with wide swath. The existed azimuth-dependent MOCO algorithms [14-18] could precisely compensate 
the azimuth-dependent motion error and modify the azimuth matched filtering function in order to eliminate the influence of azimuth-variant motion error.

Based on the signal model in [3], we investigate the cause of RCMC error as well as its definite expression deduction for MMW SAR system in this paper. A background assumption is confirmed that the trajectory information is accurately recorded by the INS and the whole motion compensation procedure is processed without autofocus step. The one-step MOCO-based imaging algorithm is investigated, which compensates rangedependent motion error before RCMC in order to suppress the residual envelope and phase error of RCM range profile. Moreover, according to the analytical expression of the residual spatial variant error, an accurate azimuth match filtering (AMF) function is modified by precise topography- and aperture-dependent motion compensation (PTA) [17], which compensates the residual azimuthdependent motion errors remained by RCMC. In this paper, the conventional two-step MOCO-based imaging algorithm is introduced for comparison, theoretical analysis to the superiority of one-step MOCO would be adequately verified by simulated and real-measured data experiments.

The whole paper is organized as follows: Section 2 gives the signal and geometry model of the SAR imaging, RCMC accuracy respect to the residual range-dependent error is analyzed as well, and flowcharts of one-step and two-step MOCO-based SAR imaging algorithms are then given for comparison. In Section 3, RCMC error comparison between one-step and two-step MOCO is discussed, and computational burden of both methods is also analyzed in detail. In Section 4, extensive experimental results are given with both simulated and real measured MMW airborne SAR data. Conclusions are given in the last section.

\section{One-step MOCO-based SAR imaging algorithm}

\subsection{SAR imaging and RCMC error analysis}

The squinted SAR geometry is given in Fig. 1. In the model above, the SAR sensor travels along a straight-line flight path with a constant velocity $v$ in ideal conditions, and the synthetic aperture length is $L$. Symbol $O$ stands for the original point of SAR sensor. The ideal linear trajectory is defined as $X$-axis corresponding to the azimuth direction, point $A$ denotes the ideal position of platform. During the data acquisition, the radar beam illuminates at squint angle $\varphi$, symbol $C$ denotes the scene center, and $r$ represents the range from $C$ to radar at squint angle $\varphi$. Symbol $P$ stands for the target located on the scene center line $O^{\prime} C$, which is parallel to the trajectory. The distance between target $P$ and scene center $C$ is given by $x$, and the distance between $O^{\prime}$ and $C$ is given by $x_{0}$. The instantaneous range from target $P$ to the radar in the conical coordinate system [19] is given by:

$R_{n}(X)=\sqrt{(r \cos \varphi)^{2}+(X-x-r \sin \varphi)^{2}}, \quad-\frac{L}{2} \leq X \leq \frac{L}{2}$

where $X=v t_{m}$ denotes the instantaneous azimuth coordinate of antenna phase center at slow time $t_{m}$. The expression of echo signal from target $P$ is given by:

$$
\begin{aligned}
S(\tau, X)= & \varepsilon_{p} \cdot \operatorname{rect}\left(\frac{\tau-\Delta t}{T_{p}}\right) \cdot \operatorname{rect}\left(\frac{X-x_{0}-x}{L}\right) \\
& \times \exp \left[j 2 \pi\left(-f_{c} \Delta t+\frac{\alpha(\tau-\Delta t)^{2}}{2}\right)\right]
\end{aligned}
$$

where $\alpha$ is the chirp rate, $T_{p}$ is the pulse duration width, $f_{c}$ is the center frequency, $\tau$ denotes the range fast-time, $\Delta t=2 R_{n}(X) / c$ stands for the round-way propagation time of electromagnetic wave between target $P$ and radar, $c$ is the speed of light. $\varepsilon_{p}$ corresponds to the complexvalued scattering amplitude of the point target, symbol rect (.) denotes the rectangular window function. According to [7], the expression of range compressed signal is given by:

$$
\begin{aligned}
S(\tau, X)= & \varepsilon_{p} \cdot \operatorname{sinc}\left[T_{p} \alpha\left(\tau-\frac{2 R_{n}(X)}{c}\right)\right] \cdot \operatorname{rect}\left(\frac{X-x_{0}-x}{L}\right) \\
& \times \exp \left[-j \frac{4 \pi}{\lambda} R_{n}(X)\right]
\end{aligned}
$$

where symbol sinc $(\cdot)$ is expressed as $\operatorname{sinc}(a)=$ $\sin (\pi a) / \pi a$. However, the actual motion of platform is not ideal, so the range compressed signal in (3) is blurred with motion error $\Delta R=A^{\prime} P-A P$. Motion compensation is then processed to the blurred range compressed signal in order to remove the envelope and phase of motion errors, so that most of the errors would be compensated. Due to our early works in [3], the signal model as well as the residual phase after deramp processing are calculated for a refined phase gradient autofocus. Based on the former accumulation, in this paper, we try to investigate the mathematical explanation of RMC error with respect to the residual motion errors remained by the range-independent MOCO. Order $\Delta R_{E}$ to represent the residual motion error remained by the range-independent MOCO before RCMC, which would induce serious envelope and phase error to RCMC, and decrease the azimuth pulse compression accuracy. The envelope part of $\Delta R_{E}$ is usually limited within a range bin in actual processing, so we ignore the small envelope of $\Delta R_{E}$. Applying the range Fourier transform (FT) with respect to $\tau$, the signal is transferred to the range wavenumber domain as shown in (4). The constant and envelope terms of signal expression could be omitted for analysis without adverse effect. 


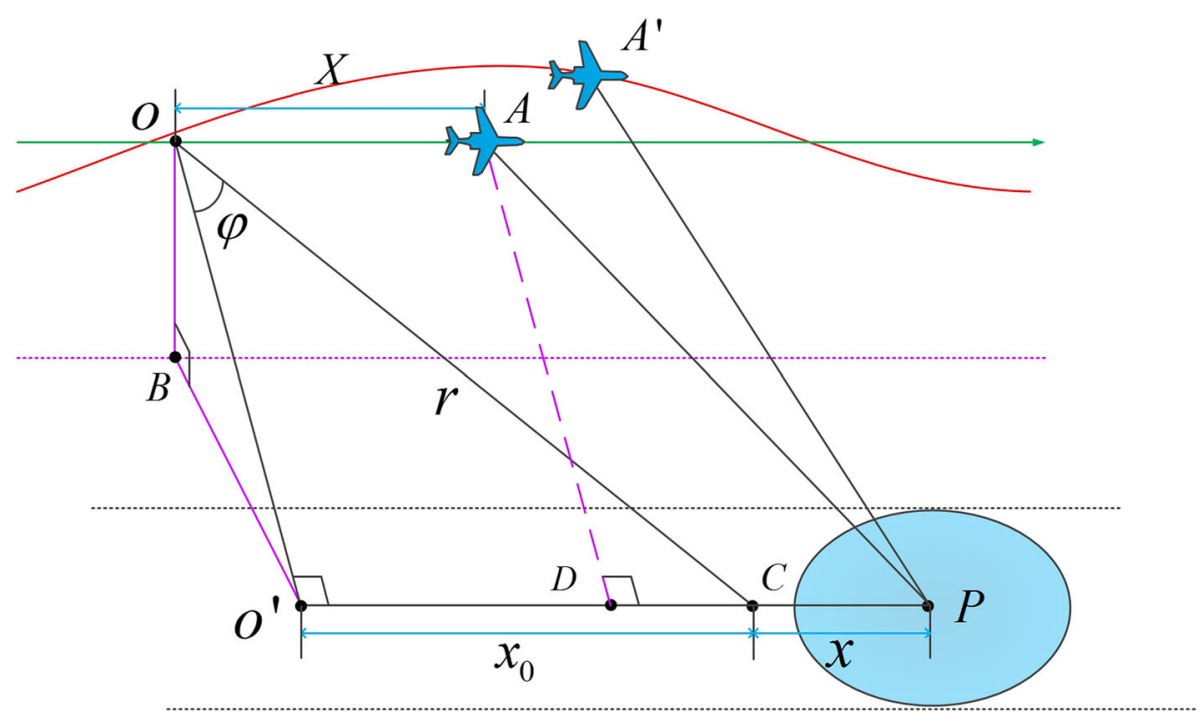

Fig. 1 Geometric model of squint SAR imaging

$$
\begin{aligned}
S\left(\Delta K_{r}, X\right)= & \int S(\tau, X) \exp \left(-j \Delta K_{r} \frac{\tau c}{2}\right) \cdot d \frac{\tau c}{2} \\
= & \exp \left\{-j K_{r} .\right. \\
& {\left.\left[\sqrt{(r \cos \varphi)^{2}+(X-x-r \sin \varphi)^{2}}+\Delta R_{E}(X)\right]\right\} }
\end{aligned}
$$

where $K_{r}$ represents the range wavenumber spectrum with $K_{r}=K_{r c}+\Delta K_{r}$ and $K_{r c}=4 \pi f_{c} / c$ is the range wavenumber center with $\Delta K_{r} \in\left[-2 \pi \alpha T_{p} / c, 2 \pi \alpha T_{p} / c\right]$. Then, applying the azimuth FT to (4) with respect to $X$, the impact of $\Delta R_{E}$ to the stationary phase point could be ignored for simplifying the analysis formula. The expression of signal in two-dimensional wavenumber domain is given by [3]:

$$
\begin{gathered}
S\left(\Delta K_{r}, K_{x}\right)=\exp \left\{-j\left[\left(\sqrt{K_{r}^{2}-K_{x}^{2}} \cos \varphi+K_{x} \sin \varphi\right) \cdot r+K_{x} \cdot x\right]\right\} \\
\times \exp \left[-K_{r} \cdot \Delta R_{E}\left(X^{*}\right)\right] \\
X^{*}=-\frac{r \cos \varphi \cdot K_{x}}{\sqrt{K_{r}^{2}-K_{x}^{2}}}+r \sin \varphi+x
\end{gathered}
$$

where $K_{x}$ represents the azimuth wavenumber spectrum and $X^{*}$ denotes the ideal stationary phase point. The first phase term of (5) is expressed by a Taylor expansion with respect to $\Delta K_{r}=K_{r}-K_{r c}$ around $\Delta K_{r}=0$, so (5) is expressed as:

$$
\begin{aligned}
S\left(\Delta K_{r}, K_{x}\right) \approx & \exp \left[-j\left(\sqrt{K_{r c}^{2}-K_{x}^{2}} r \cos \varphi+K_{x}(r \sin \varphi+x)\right)\right] \\
& \times \exp \left(-j \frac{K_{r c} r \cos \varphi}{\sqrt{K_{r c}^{2}-K_{x}^{2}}} \Delta K_{r}\right) \cdot \exp \left[j \frac{K_{x}^{2} r \cos \varphi}{2\left(K_{r c}^{2}-K_{x}^{2}\right)^{\frac{3}{2}}} \Delta K_{r}^{2}\right] \\
& \times \exp \left[-K_{r} \cdot \Delta R_{E}\left(X^{*}\right)\right]
\end{aligned}
$$

where the second term of (7) represents the range cell migration and the third term represents the second-order coupling phase. One may multiply both sides of Eq. (7) by $H_{\mathrm{RCMC}}\left(K_{x}, K_{r}\right)$ and $H_{\mathrm{SORC}}\left(K_{x}, K_{r}\right)$ to perform the RCMC and second-order range compression (SORC).

$$
\begin{aligned}
& H_{\mathrm{RCMC}}\left(K_{x}, K_{r}\right)=\exp \left[j \Delta K_{r}\left(\frac{K_{r c} r \cos \varphi}{\sqrt{K_{r c}^{2}-K_{x}^{2}}}-r\right)\right] \\
& H_{\mathrm{SORC}}\left(K_{x}, K_{r}\right)=\exp \left[-j \frac{K_{x}^{2} r \cos \varphi}{2\left(K_{r c}^{2}-K_{x}^{2}\right)^{\frac{3}{2}}} \Delta K_{r}^{2}\right]
\end{aligned}
$$

The expression of signal in two-dimensional wavenumber domain processed by RCMC and SORC is given as follows:

$$
\begin{aligned}
S\left(\Delta K_{r}, K_{x}\right)= & \exp \left\{-j\left[K_{x}(x+r \sin \varphi)+\Delta K_{r} r\right.\right. \\
& \left.\left.\quad+r \cos \varphi \sqrt{K_{r c}^{2}-K_{x}^{2}}\right]\right\} \\
\times & \exp \left[-j K_{r} \cdot \Delta R_{E}\left(X^{*}\right)\right]
\end{aligned}
$$

where the second term of (10) is shown in (11), which is considered the residual motion error phase in twodimension wavenumber domain, decreasing the accuracy of RCMC seriously.

$$
\Phi\left(\Delta K_{r}, K_{x}\right)=\exp \left[-j K_{r} \Delta R_{E}\left(X^{*}\right)\right]
$$

Then, the relationship between $\Phi\left(\Delta K_{r}, K_{x}\right)$ and RCMC error would be theoretically analyzed. It could be observed in (6) that the residual motion error $\Delta R_{E}\left(X^{*}\right)$ is a function of range wavenumber spectrum $\Delta K_{r}$, so we may expand the second phase term in (10) by a Taylor expansion with respect to $\Delta K_{r}$ around $\Delta K_{r}=0$, which 
is expressed as follows, remaining the constant term and first-order term:

$$
\left.\Delta R_{E}\left(X^{*}\right) \approx \Delta R_{E}\left(X^{*}\right)\right|_{\Delta K_{r}=0}+\left.\frac{\partial \Delta R_{E}\left(X^{*}\right)}{\partial \Delta K_{r}}\right|_{\Delta K_{r}=0} \Delta K_{r}
$$

where

$$
\begin{aligned}
\frac{\partial \Delta R_{E}\left(X^{*}\right)}{\partial \Delta K_{r}} & =\frac{\partial X^{*}}{\partial \Delta K_{r}} \cdot \frac{\partial \Delta R_{E}\left(X^{*}\right)}{\partial X^{*}} \\
& =\frac{K_{x} \cdot\left(K_{r c}+\Delta K_{r}\right) r \cos \varphi}{\left[\left(K_{r c}+\Delta K_{r}\right)^{2}-K_{x}^{2}\right]^{\frac{3}{2}}} \cdot \frac{\partial \Delta R_{E}\left(X^{*}\right)}{\partial X^{*}}
\end{aligned}
$$

Ignoring the impact to the stationary phase point made by $\Delta R_{E}\left(X^{*}\right)$, we apply azimuth inverse Fourier transform (IFT) to (10), and we have:

$$
\begin{aligned}
S\left(\Delta K_{r}, X\right)= & \int S\left(\Delta K_{r}, K_{x}\right) \cdot \exp \left[j K_{x} X\right] \cdot d K_{x} \\
= & \exp \left[-j K_{r c}\left(\sqrt{(r \cos \varphi)^{2}+(X-x-r \sin \varphi)^{2}}\right)\right] \\
& \times \exp \left[\Delta R_{E}\left(K_{x}^{*}\right) \cdot\left(K_{r c}+\Delta K_{r}\right)\right]
\end{aligned}
$$

where the stationary phase point $K_{x}^{*}$ is given by:

$$
K_{x}^{*} \approx-K_{r c} \frac{X-r \sin \varphi-x}{\sqrt{(\cos \varphi \cdot r)^{2}+(X-x-r \sin \varphi)^{2}}}
$$

Substituting (15) into (12) with replacement of $K_{x}$ by $K_{x}^{*}$ and ordering $X-x=0$ for simply analyzing, the residual motion error in azimuth time and range wavenumber domain is given by:

$$
\Delta R_{E}(X)=\Delta R_{E}+\frac{r \sin \varphi}{K_{r c} \cos ^{2} \varphi} \cdot \frac{\partial \Delta R_{E}}{\partial X} \cdot \Delta K_{r}
$$

Substituting (16) into (14), the expression of signal is translated to the following equation:

$$
\begin{aligned}
S\left(\Delta K_{r}, X\right)= & \exp \left[-j K_{r c}\left(\sqrt{(r \cos \varphi)^{2}+(X-x-r \sin \varphi)^{2}}\right)\right] \\
& \times \exp \left[-j\left(\vartheta_{0}+\vartheta_{1} \Delta K_{r}+\vartheta_{2} \Delta K_{r}^{2}\right)\right]
\end{aligned}
$$

where the second term of (17) is the RCMC error with respect to the residual motion error $\Delta R_{E}$. This phase term is expressed as a series of $\Delta K_{r}$, so the phase and envelope component would be distinguished where

$$
\begin{aligned}
& \vartheta_{0}=K_{r c} \Delta R_{E} \\
& \vartheta_{1}=\Delta R_{E}+\frac{r \sin \varphi}{\cos ^{2} \varphi} \cdot \frac{\partial \Delta R_{E}}{\partial X} \\
& \vartheta_{2}=\frac{r \sin \varphi}{K_{r c} \cos ^{2} \varphi} \cdot \frac{\partial \Delta R_{E}}{\partial X}
\end{aligned}
$$

It is worthy to note that $\vartheta_{0}$ represents the phase component of RCMC error and $\vartheta_{1}$ denotes the envelope error of RCMC, which seriously destroys the imaging performance. $\vartheta_{2}$ is the second-order term, which slightly reflects the focusing performance in range, and the effect could be ignored in most cases.

\subsection{Flowcharts of one-step and two-step MOCO-based imaging algorithms}

As shown in Fig. 2, a flowchart of the one-step MOCObased imaging algorithm is given in this subsection, while the conventional two-step MOCO-based imaging algorithm is also given for comparison. It is here needed to explain that the flowchart is displayed in such form for the purpose of distinguishing the procedure of the one-step MOCO and two-step MOCO more legible. We introduce PTA for azimuth-variant $\mathrm{MOCO}$ in order to eliminate the influence caused by other residual specialdependent errors. In a one-step procedure, bulk envelope compensation is processed in range wavenumber domain by multiplying $H_{\mathrm{MOCO}}\left(\Delta K_{r}, X\right)$ to the range compressed data. The envelope compensation function is expressed as:

$$
H_{\mathrm{MOCO}}\left(\Delta K_{r}, X\right)=\exp \left[-j \Delta R_{r}\left(r_{s}\right) \Delta K_{r}\right]
$$

where $\Delta R_{r}$ denotes the slant range projection of motion error, which is with respect to slant range $r$ and would be expressed in (26). Then, the range-dependent motion error is compensated in range time domain by multiplying $H_{\mathrm{MOCO}}(r, X)$, where

$$
H_{\mathrm{MOCO}}(r, X)=\exp \left[-j K_{r c} \Delta R_{r}(r, X)\right]
$$

The compensated data is then transformed into two-dimensional wavenumber domain, and SORC and RCMC are processed by multiplying $H_{\mathrm{SORC}}\left(K_{x}, K_{r}\right)$ and $H_{\mathrm{RCMC}}\left(K_{x}, K_{r}\right)$, shown in (8) and (9), respectively. One-step $\mathrm{MOCO}$ has compensated most of the rangedependent motion errors, but the residual azimuthdependent motion error is remained after RCMC, which decreases the accuracy of azimuth pulse compression. Due to the residual azimuth-dependent motion error, $\vartheta_{0}$ is calculated in (18), and a method of PTA is introduced to compensate $\vartheta_{0}$ by modifying the AMF function. According to [17], the modified AMF function $H_{\mathrm{AMF}}\left(K_{x}, r\right)$ is expressed as:

$$
\begin{aligned}
H_{\mathrm{AMF}}\left(K_{x}, r\right)= & \exp \left[j K_{r c} \Delta R_{E}\left(X_{e}^{*}\right)\right] \cdot \exp \left[j K_{r c} R_{n}\left(r, X_{e}^{*}\right)\right. \\
& \left.+j K_{x}\left(X_{e}^{*}-x\right)\right]
\end{aligned}
$$

where $X_{e}^{*}$ is calculated by

$$
\frac{\partial R_{n}\left(X_{e}^{*}\right)}{\partial X_{e}^{*}}+\frac{\partial \Delta R_{E}\left(X_{e}^{*}\right)}{\partial X_{e}^{*}}+\frac{K_{x}}{K_{r c}}=0
$$

As the one-step MOCO-based imaging algorithm is described in detail, we briefly describe the procedure of the two-step MOCO-based imaging algorithm for comparison. For two-step MOCO, the envelope compensation and the first-phase compensation are processed with respect to the reference slant range $r_{s}$ before $\mathrm{RCMC}$, and the second range-dependent MOCO step is 


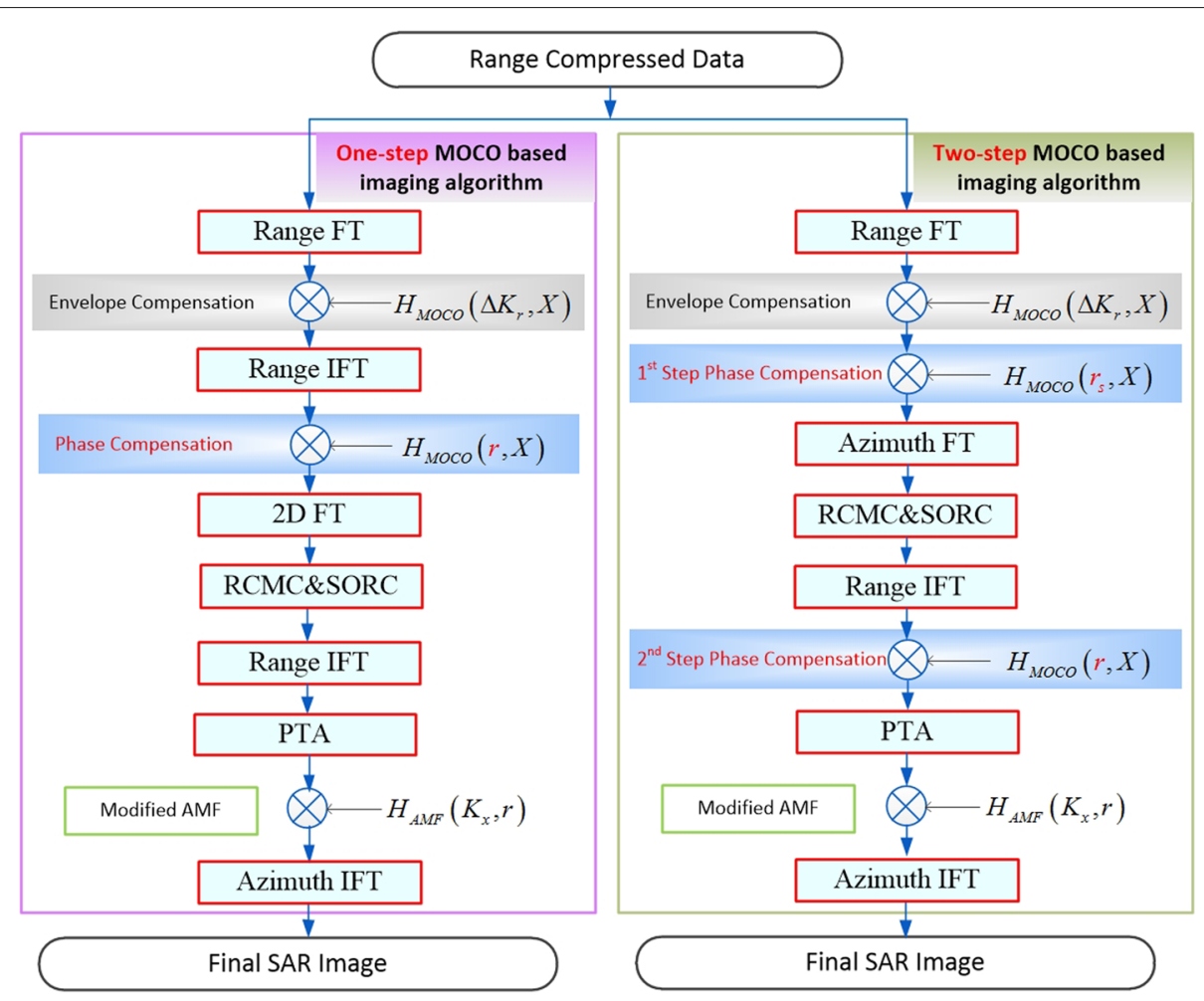

Fig. 2 Flowchart of one-step MOCO-based imaging algorithm compared with conventional two-step MOCO-based imaging algorithm

processed after RCMC, but serious RCM envelope error is remained at this step. In order to make the comparison more equitable, azimuth-dependent motion error is then compensated by method of PTA.

\section{Comparative analysis of one-step and two-step MOCO}

\subsection{RCMC error comparison between one-step and two-step MOCO}

In the previous section, we analyze the RCMC error with respect to the residual motion error $\Delta R_{E}$, but $\Delta R_{E}$ is different for the one-step and two-step MOCO. In this subsection, we focus on calculating $\Delta R_{E}$ and comparing the RCMC error between the one-step and two-step MOCO.

The actual range history at slant range $r$ is expressed as:

$\tilde{R}(r, X)=\sqrt{(X-x-r \sin \varphi-\Delta x)^{2}+\left(Y_{t}-\Delta y\right)^{2}+(H-\Delta z)^{2}}$

where $\Delta x, \Delta y$, and $\Delta z$ represent the along track error, cross track error, and height error, respectively. $X_{t}=$ $r \sin \varphi$ denotes the projection of $r$ on $x$ direction, $Y_{t}=\sqrt{r^{2} \cos ^{2} \varphi-H^{2}}$ denotes the projection of $r$ on $y$ direction, and $H$ is the height of the platform. The azimuth-independent motion error respect to range $r$ is expressed as:

$$
\Delta R_{r}(r)=\frac{X_{t}}{r} \Delta x+\frac{Y_{t}}{r} \Delta y+\frac{H}{r} \Delta z
$$

For the two-step MOCO algorithm, the rangeindependent component of motion error is compensated in the first step. The residual motion error $\Delta \tilde{R}_{E}$ contains range-dependent component and azimuth-dependent component, which is given by:

$$
\begin{aligned}
& \Delta \tilde{R}_{E}(r, X)=\tilde{R}(r, X)-R_{n}(r, X)-\Delta R_{r}\left(r_{s}\right) \\
& =\frac{r_{s}-r}{r_{s}^{2}} \cdot\left(-\frac{H^{2}}{\sqrt{r_{s}^{2} \cos ^{2} \varphi-H^{2}}} \Delta y+H \Delta z\right)+\delta R_{a}(X)
\end{aligned}
$$

where $r$ denotes the range bin of target, $r_{s}$ denotes the slant range from the radar to the beam center, $\delta R_{a}$ is the azimuth-dependent motion error. It is obvious in (27) that the residual motion error is in proportion to the range between target and scene center, so the error is diffused along range direction.

For one-step MOCO, range-dependent motion error is compensated before RCMC. However, a new error $\delta R_{o}$ is introduced for squint SAR, the residual motion error before RCMC is given by: 

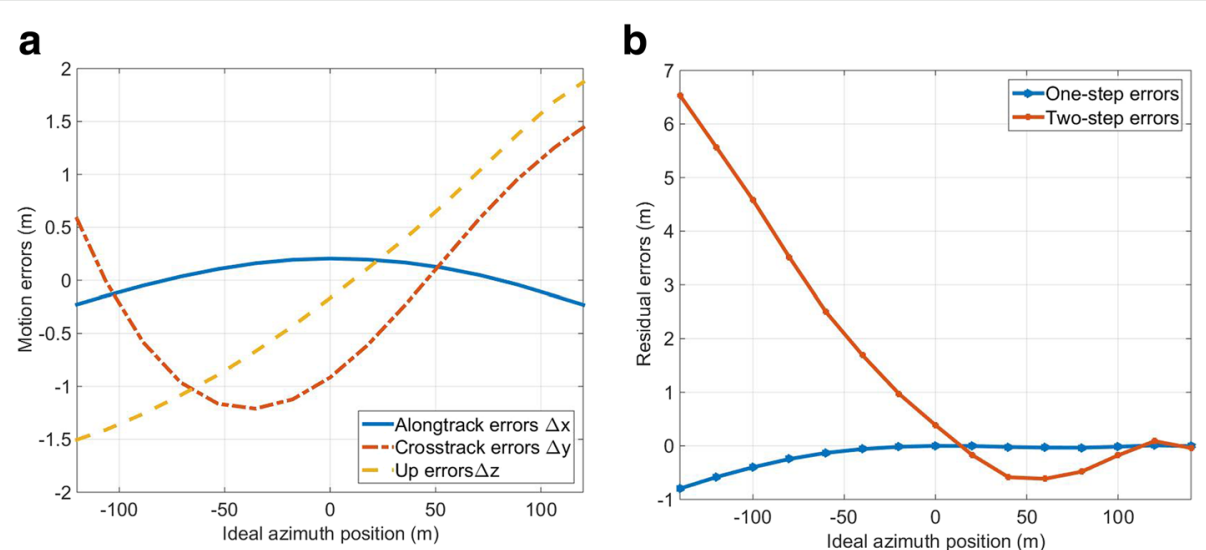

Fig. 3 Real measured motion error and corresponding residual RCMC envelope error. a Real-measured motion error. b Corresponding residual RCMC envelope error

$$
\begin{aligned}
\Delta R_{E}(X)= & \delta R_{o}(X)+\tilde{R}(r, X)-R_{n}(r, X)-\Delta R_{r}(r) \\
= & \delta R_{o}(X)+\delta R_{a}(X) \\
\approx & -\frac{(X-x) \sin \varphi}{r_{s}^{2}} \cdot\left(-\frac{H^{2}}{\sqrt{r_{s}^{2} \cos ^{2} \varphi-H^{2}}} \Delta y+H \Delta z\right) \\
& +\delta R_{a}(X)
\end{aligned}
$$

where $x$ denotes the azimuth position of the target. In this section, we mainly focus on providing the comparison of RCMC envelope error between the two-step and one-step MOCO, which is expressed as $\vartheta_{1}$ in (19). Order

$$
u(X)=-\frac{H^{2}}{\sqrt{r_{s}^{2} \cos ^{2} \varphi-H^{2}}} \Delta y+H \Delta z
$$

Substituting (29) into (19), the RCMC envelope error for the two-step MOCO is given by:

$$
\Delta T_{1} \approx \frac{r_{s}-r}{r^{2}}\left[u(X)+\frac{r \sin \varphi}{\cos ^{2} \varphi} \cdot u^{\prime}(X)\right]
$$

Table 1 Simulation parameters

\begin{tabular}{ll}
\hline Carrier frequency & $35 \mathrm{GHz}$ \\
Pulse repetition frequency & $2000 \mathrm{~Hz}$ \\
Velocity & $70 \mathrm{~m} / \mathrm{s}$ \\
Pulse width & $2 \mu \mathrm{s}$ \\
Center closest slant range & $4000 \mathrm{~m}$ \\
Squint angle & $5^{\circ}$ \\
Grazing angle & $45^{\circ}$ \\
Range resolution & $0.15 \mathrm{~m}$ \\
Azimuth resolution & $0.15 \mathrm{~m}$ \\
Point A coordinate & $(0,400)$ \\
Point B coordinate & $(0,0)$ \\
Point C coordinate & $(0,-400)$ \\
\hline
\end{tabular}

Similarly, substituting (30) into (19), the RCMC envelope error for the one-step MOCO is given by:

$$
\Delta T_{2} \approx \frac{(X-x) \sin \varphi}{r^{2}}\left[u(X)+\frac{r \sin \varphi}{\cos ^{2} \varphi} \cdot u^{\prime}(X)\right]
$$

where the azimuth-dependent motion error $\delta R_{a}$ has little impact on the RCMC envelope, so it is not considered in this paper. The ratio between $\Delta T_{1}$ and $\Delta T_{2}$ is expressed as:

$$
\frac{\Delta T_{1}}{\Delta T_{2}} \approx\left|\frac{r_{s}-r}{(X-x) \sin \varphi}\right|
$$

where the numerator of (32) represents the range difference between target and scene center and the denominator represents the length of range walk. It's clear that the RCMC envelope error is in proportion to the range difference for two-step MOCO, so the imaging performance would be seriously destroyed at the points far from scene center line. However, in some cases, the effect of residual range-dependent motion error could be neglected if the maximum of RCMC envelope is within $1 / 4$ rang bin, which is shown as:

$$
\max \left|\Delta R_{E}+\frac{r \sin \varphi}{\cos ^{2} \varphi} \cdot \frac{\partial \Delta R_{E}}{\partial X}\right| \leq \frac{1}{4} \Delta r
$$

where $\max |\cdot|$ denotes the maximum of the absolute value. For the two-step MOCO, the inequality becomes:

$$
\max \left|\frac{r_{s}-r}{r^{2}}\left[u(X)+\frac{r \sin \varphi}{\cos ^{2} \varphi} \cdot u^{\prime}(X)\right]\right| \leq \frac{1}{4} \Delta r
$$

And for the one-step MOCO, the inequality becomes:

$$
\max \left|\frac{(X-x) \sin \varphi}{r^{2}}\left[u(X)+\frac{r \sin \varphi}{\cos ^{2} \varphi} u^{\prime}(X)\right]\right| \leq \frac{1}{4} \Delta r
$$




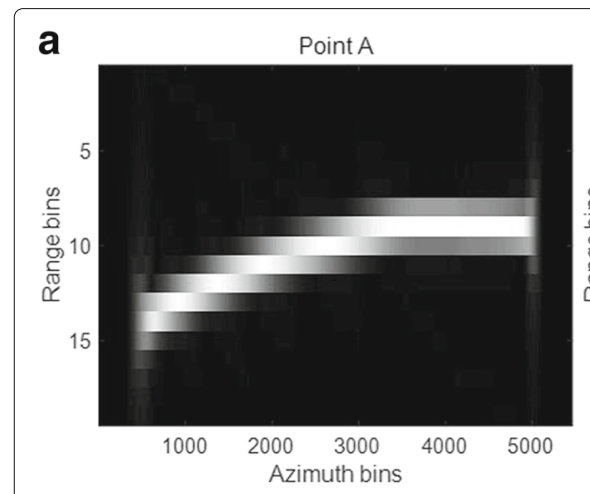

b

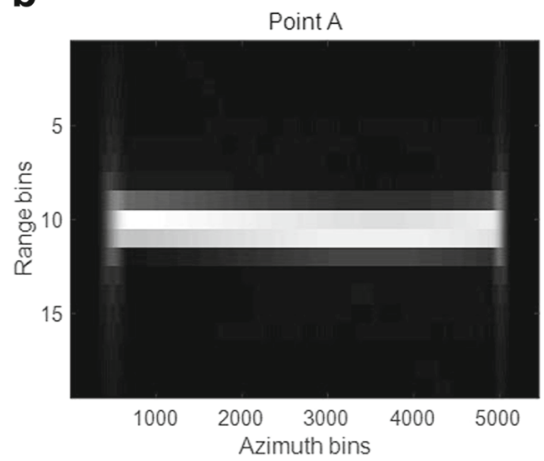

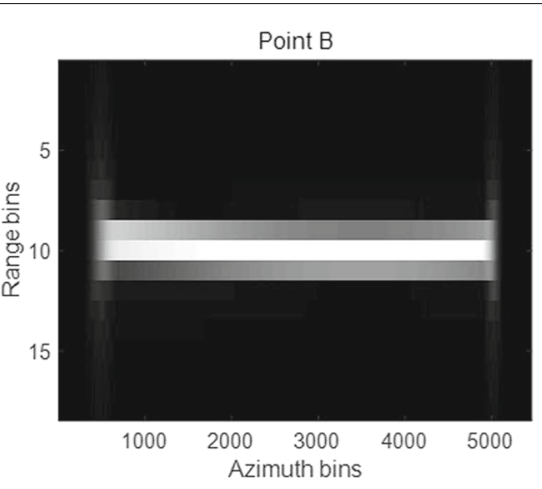

Point B

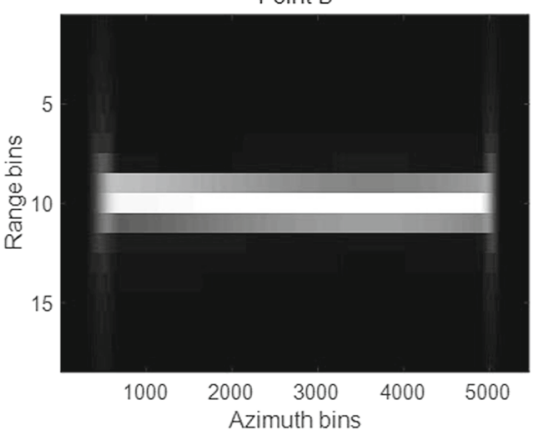

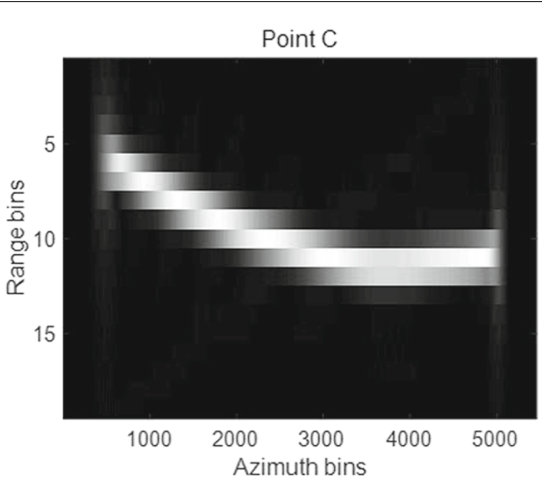

Point C

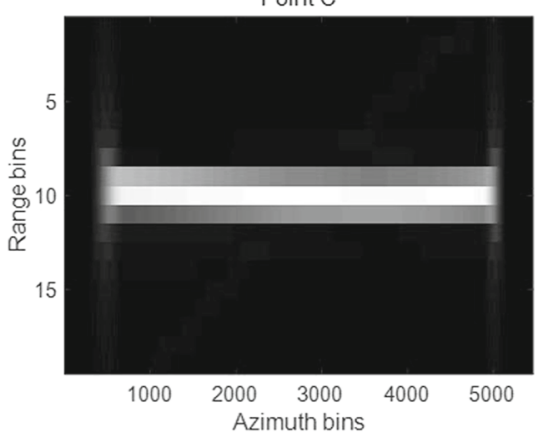

Fig. 4 Comparison of the two-step MOCO and one-step MOCO in RCMC results of points A, B and C. a Two-step MOCO results. b One-step MOCO results
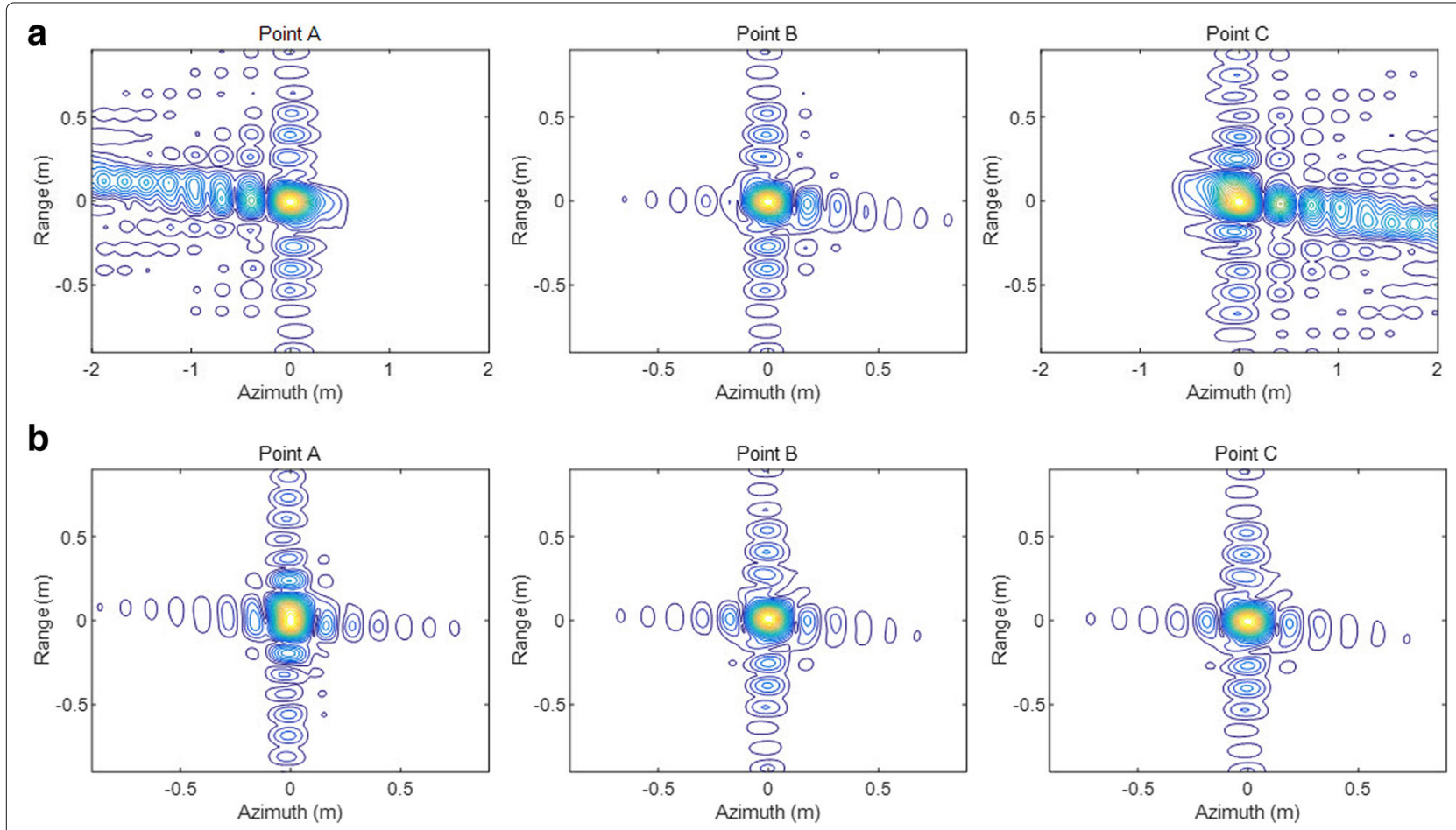

Fig. 5 Comparison of the two-step MOCO and one-step MOCO in imaging results of points A, B, and C. a Two-step MOCO results. b One-step MOCO results 


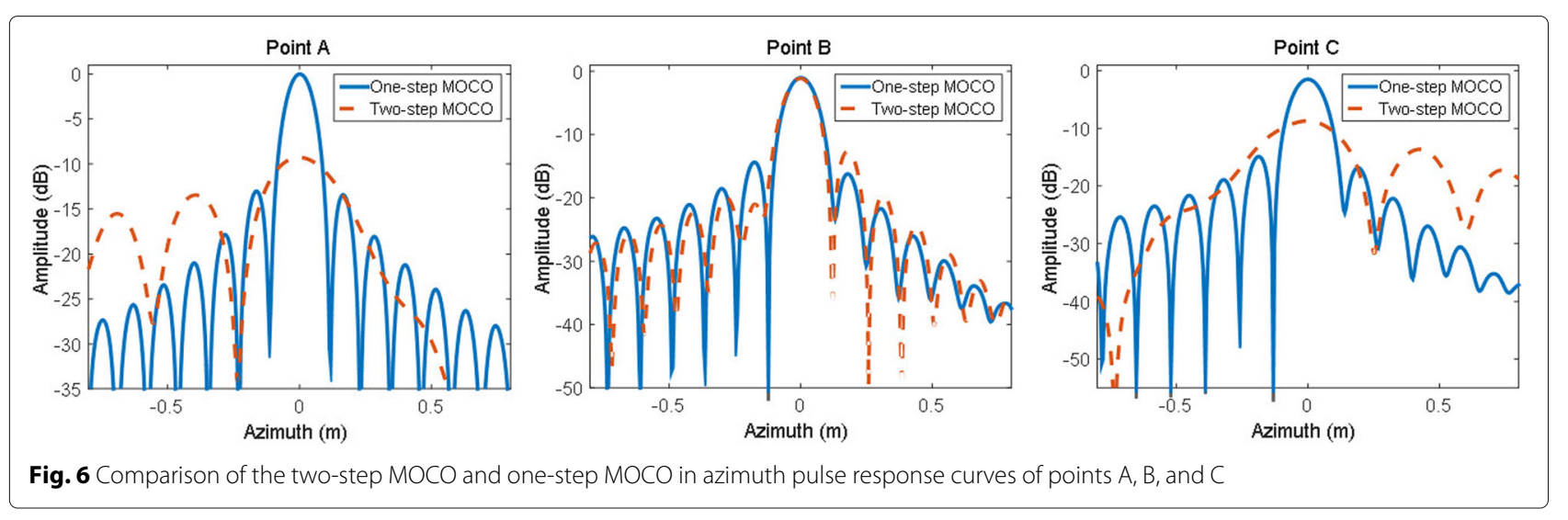

It could be found that, for SAR imaging with wide swath, the requirement of inequation (34) is hard to satisfy at points far from the scene center line, while inequation (35) is easier to meet. A simulation result of the RCM error comparison with real-measured trajectory deviations between the two-step and one-step MOCO is shown in Fig. 3. The trajectory deviations are extracted from a real position and orientation system shown in Fig. 3a. The residual RCMC envelope error is calculated with a squint angle of $5^{\circ}$ and a pitch angle of $45^{\circ}$, the center closest slant range is $4000 \mathrm{~m}$, and the range difference between target and scene center is $100 \mathrm{~m}$. The corresponding residual RCMC envelope errors are shown in Fig. 3b. It is clear that the envelope error of the two-step MOCO is up to several meters, while the one-step MOCO significantly overcomes this problem.

\subsection{Computational burden analysis}

In this subsection, the computational burden of the one-step and two-step MOCO-based SAR imaging algorithms is respectively measured by operating number of fast Fourier transform (FFT), inverse fast Fourier transform (IFFT), and complex multiplication for comparison. As shown in Fig. 2, suppose the azimuth and range point numbers are denoted by $N_{a}$ and $N_{r}$. It needs to be noticed that we analyze the operand by merging adjoining phase terms and without regard of the calculation of PTA operation. For the one-step MOCObased imaging algorithm, there are $4 N_{a}$ times $N_{r}$-point FFT/IFFT operators, $2 N_{r}$ times $N_{a}$-point FFT/IFFT operators, and 5 times $N_{r} \times N_{a}$-point complex multiplications to obtain a focused imaging. Comparing with the conventional two-step based imaging algorithm, there are $2 N_{a}$ times $N_{r}$-point FFT/IFFT operators, $2 N_{r}$ times $N_{a}$-point FFT/IFFT operators, and 5 times $N_{r} \times N_{a}$-point complex multiplications. It could be found that the one-step MOCO-based imaging algorithm adds $2 N_{r}$ more times $N_{a}$-point FFT/IFFT operators, which slightly increases the computational burden and exchanges for a better focused imagery.

\section{Simulated and real data experiments}

\subsection{Experiments with simulated data}

In this subsection, a group of point target simulation experiments are set to demonstrate the effectiveness of the one-step MOCO-based imaging algorithm compared with the two-step MOCO-based imaging algorithm. Simulation parameters and three-point target coordinates are shown in Table 1. It needs to be emphasized that points A and $C$ are far from the scene center line, while point $B$ lies on the scene center line. In this simulation experiment, the performance of the one-step MOCO imaging algorithm is validated by simulated data mixed with motion errors which are extracted from airborne INS, which are shown in Fig. 3a.

At first, we present the comparison of RCMC error with respect to the residual range-dependent motion error between the two-step MOCO and one-step MOCO. In order to illustrate the RCMC error explicitly, the range profile of three points are shown in two-dimensional time domain. It could be easily found in Fig. 4a that the residual RCMs of points $\mathrm{A}$ and $\mathrm{C}$ are obvious, except for point $\mathrm{B}$ because it is lying on the scene center line without range-dependent motion error before RCMC. These RCMC errors are deemed to coincide well

Table 2 Focusing performance comparison between two focusing algorithms

\begin{tabular}{lllll}
\hline Target point & Method & PSLR $(\mathrm{dB})$ & ISLR (dB) & IRW (m) \\
\hline A & One-step MOCO & -13.0416 & -10.4187 & 0.1444 \\
& Two-step MOCO & -4.1975 & -4.4064 & 0.3631 \\
B & One-step MOCO & -13.3593 & -11.3825 & 0.1531 \\
& Two-step MOCO & -11.5402 & -11.1442 & 0.1575 \\
C & One-step MOCO & -13.4320 & -11.4704 & 0.1663 \\
& Two-step MOCO & -4.9471 & -5.0134 & 0.3894 \\
\hline
\end{tabular}


Table 3 Experiment parameters

\begin{tabular}{ll}
\hline Carrier frequency & $35 \mathrm{GHz}$ \\
Pulse repetition frequency & $5000 \mathrm{~Hz}$ \\
Velocity & $70 \mathrm{~m} / \mathrm{s}$ \\
Pulse width & $20 \mu \mathrm{s}$ \\
Center closest slant range & $4000 \mathrm{~m}$ \\
Squint angle & $5^{\circ}$ \\
Grazing angle & $45^{\circ}$ \\
Range resolution & $0.15 \mathrm{~m}$ \\
Azimuth resolution & $0.15 \mathrm{~m}$ \\
\hline
\end{tabular}

with the expression of $\vartheta_{1}$ in (19). It is also shown in Fig. 4a that the residual RCMC errors of points $\mathrm{A}$ and $\mathrm{C}$ processed by two-step $\mathrm{MOCO}$ are spanning across several range cells, so the focusing performance would be seriously damaged. The RCM corrected range profiles of points $\mathrm{A}, \mathrm{B}$, and $\mathrm{C}$ processed by the one-step MOCO are shown in Fig. 4b for comparison, while all the RCMC errors of points $A, B$, and $C$ are well removed.

Then, azimuth compression performance of the onestep MOCO-based imaging algorithm is investigated. The two-dimensional images of points $\mathrm{A}, \mathrm{B}$, and $\mathrm{C}$ processed by the conventional two-step MOCO-based imaging algorithm and one-step $\mathrm{MOCO}$-based imaging algorithm are shown in Fig. 5. Because of the existence of residual RCMC errors, the imaging algorithm based on the conventional two-step MOCO fails to focus in azimuth for points $\mathrm{A}$ and $\mathrm{C}$, as shown in Fig. 5a. While the wellfocused imaging results of points $A, B$, and $C$ processed by the one-step MOCO-based imaging algorithm are shown in Fig. 5b. Figure 6 shows the comparison of azimuth point spreading response of points A, B, and C. In order to evaluate the focused improvement of the proposed algorithm comparing with the conventional one, three quantitative metrics are introduced to measure the point impulse responses of points $\mathrm{A}, \mathrm{B}$, and $\mathrm{C}$, which are shown in Table 2. The quantitative metrics are peak side-lobe ratio, (PSLR), integrated side-lobe ratio (ISLR), and impulse response width (IRW). It is obvious that the focusing degradation of the conventional two-step MOCO-based imaging algorithm is overcame by the one-step MOCObased imaging algorithm, especially for points $\mathrm{A}$ and $\mathrm{C}$, which are far from the scene center line.

\subsection{Experiments with real-measured data}

In this subsection, a set of comparison experiment is provided based on the processing of measured data recorded by an experimental airborne MMW SAR system. The instantaneous position and motion parameters of platform are measured by a high-accuracy INS equipped on the platform. Detailed radar parameters are shown in Table 3. An imaging result processed by the onestep MOCO-based imaging algorithm is shown in Fig. 7. In order to compare the imaging performance between the conventional two-step MOCO-based imaging algorithm and one-step MOCO-based imaging algorithm more vividly, two typical areas named scene 1 and scene 2 with obvious point-like targets are highlighted by the yellow rectangles in the scene. The picked two scenes are respectively magnified in Fig. 8a, b for imaging performance comparison, where the two-step MOCO imaging results are lied on the left, while the one-step MOCO imaging results are lied on the right. It is shown that defocusing of targets in the images is distinct for the twostep MOCO-based imaging algorithm, and the one-step

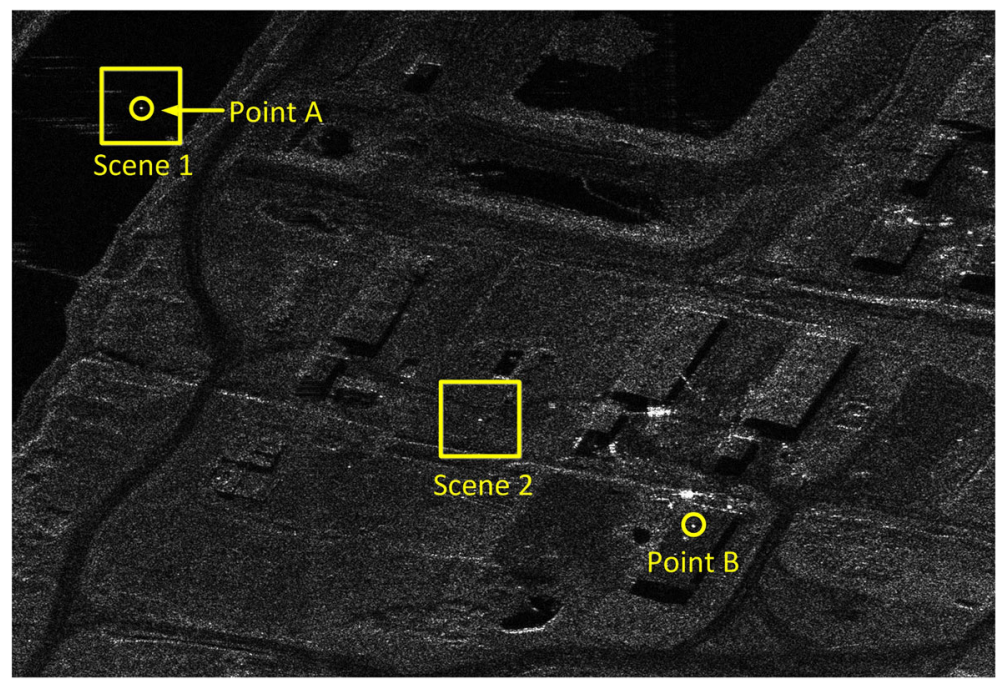

Fig. 7 Imaging result processed by the one-step MOCO-based imaging algorithm 

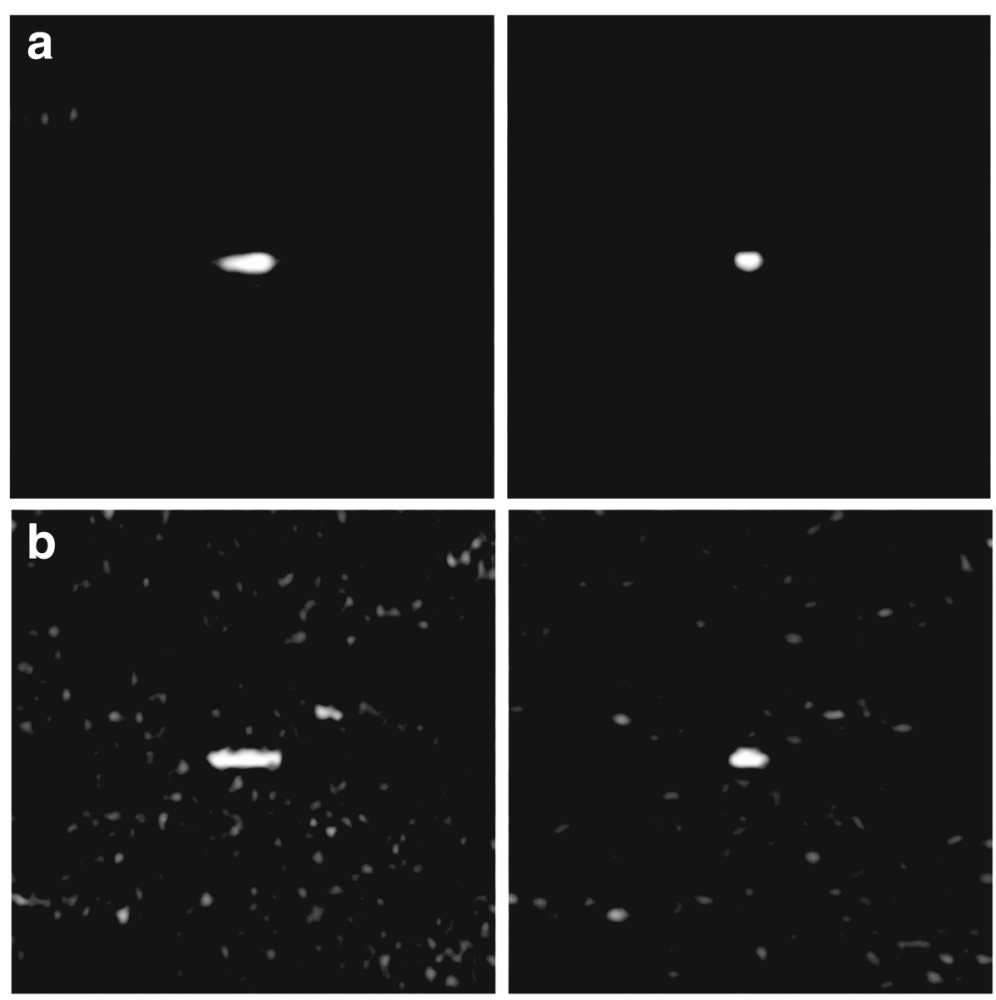

Fig. 8 Comparison of the two-step MOCO-based imaging algorithm and one-step MOCO-based imaging algorithm in local amplification results of scene 1 and scene 2. a Comparison of scene 1 (the two-step MOCO-based imaging result is on the left, the one-step MOCO-based imaging result is on the right). $\mathbf{b}$ Comparison of scene 2 (two-step MOCO-based imaging result is on the left, the one-step MOCO-based imaging result is on the right)

MOCO significantly removes the RCMC error, so the targets are well focused. Moreover, in order to check the azimuth point spreading response improvement of the one-step MOCO-based imaging algorithm, two isolated point-like targets named point $A$ and point $B$ are extracted from Fig. 7 by yellow circle for azimuth point spreading response function comparison. The comparison results of point $A$ and point $B$ are shown in Fig. 9a, b, respectively. A better azimuth pulse response function could be obtained by the one-step MOCO-based imaging algorithm, compared with the two-step. The quantitative analysis results of the azimuth point spreading response functions of Fig. 9 are listed in Table 4. It could be found in the experiments above that, focusing performance is sensitive to the residual RCMC error especially for high-resolution MMW SAR imaging, so conventional two-step MOCObased imaging algorithm is not suitable for this case. The one-step MOCO removes the range-dependent motion
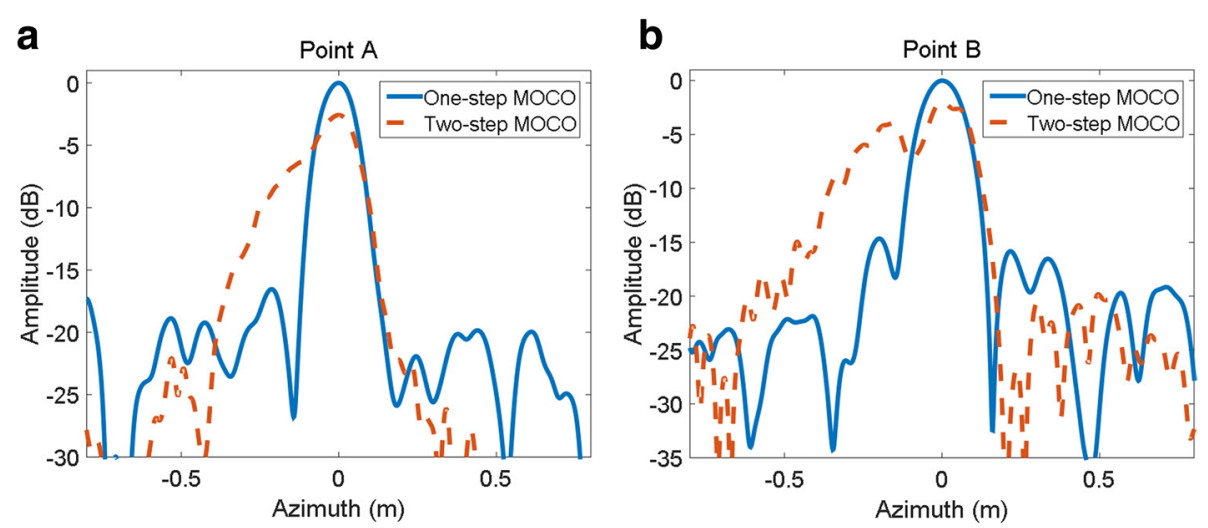

Fig. 9 Azimuth point spreading response function comparison of A and B. a Point A. b Point B 
Table 4 Focusing performance comparison of points $A$ and $B$ between two focusing algorithms

\begin{tabular}{lllll}
\hline Target point & Method & PSLR (dB) & ISLR (dB) & IRW (m) \\
\hline A & One-step MOCO & -16.5387 & -10.7167 & 0.1575 \\
& Two-step MOCO & -15.5048 & -8.6454 & 0.2975 \\
B & One-step MOCO & -14.6624 & -10.6820 & 0.1702 \\
& Two-step MOCO & -0.6995 & 2.3087 & 0.3755 \\
\hline
\end{tabular}

error before RCMC, so that the range profile after RCMC is accurate.

In order to verify the calculation analysis in Section 2, we record the calculation time for both two-step and one-step MOCO-based SAR imaging algorithms. The computer platform is installed with Windows10 64-bit operating system, Core i7-4720HQ@2.6GHz CPU, 16-GB memory and Matlab with version of R2015a. A block of $16,384 \times 8192$ (range $\times$ azimuth) points SAR data is used for test, the whole data is divided into four sub-blocks in azimuth, and the calculation time of the two-step and one-step MOCO-based imaging algorithms are 855.13s and 909.49 s, respectively. With the nearly equal computation complexity compared with the two-step MOCO, the one-step MOCO is applicative for practical MMW SAR imaging application.

\section{Conclusions}

The conventional two-step MOCO algorithm remains the range-dependent motion error before RCMC, which decreases the accuracy of RCMC in two-dimensional wavenumber domain, inducing serious envelope and phase error to the range profile. In this paper, analytical expressions of these errors are deduced in detail. The onestep MOCO-based imaging algorithm is also investigated to compare with the conventional two-step MOCO-based imaging algorithm, which removes the range-dependent motion error before RCMC, so the RCMC error is significantly suppressed. Simulations and measured MMW data experiments illustrate the outperforms of the onestep MOCO-based SAR imaging algorithm, which verify the analysis in this paper.

\section{Acknowledgements}

The authors thank the anonymous reviewers for their valuable comments to improve the paper quality. This work was supported by the National Natural Science Foundation of China under grant numbers 61301280 and 61301293 and the High-Resolution Earth Observation System Major Special Project Youth Innovation Foundation of China under grant number GFZX04060103.

\section{Competing interests}

The authors declare that they have no competing interests.

\section{Author details}

${ }^{1}$ National Lab of Radar Signal Processing and the Collaborative Innovation Center of Information Sensing and Understanding, Xidian University, Xi'an 710071, People's Republic of China. ${ }^{2}$ Beijing Institute of Radio Measurement,
The Second Academy of China Aerospace Science and Industry Corporation (CASIC), Beijing 100854, People's Republic of China.

Received: 13 May 2016 Accepted: 28 October 2016

Published online: 10 November 2016

References

1. MD Xing, XW Jiang, RB Wu, et al, Motion compensation for UAV SAR based on raw radar data. IEEE Trans. Geosci. Remote Sensing. 47(8), 2870-2883 (2009)

2. AE Azouz, ZF Li, Improved phase gradient autofocus algorithm based on segments of variable lengths and minimum-entropy phase correction. IET Radar Sonar Navigation. 9(4), 467-479 (2015)

3. L Zhang, MD Xing, ZQ Qiao, Wavenumber-domain autofocusing for highly squinted UAV SAR imagery. IEEE Sensors J. 12(5), 1574-1588 (2012)

4. A Moreira, YH Huang, Airborne SAR processing of highly squinted data using a chirp scaling approach with integrated motion compensation. IEEE Trans. Geosci. Remote Sensing. 32(5), 1029-1040 (1994)

5. WG Carrara, RM Majewshi, RS Goodman, Spotlight Synthetic Aperture Radar Signal Processing Algorithm. (Artech House, Boston, 1995), pp. 15-27

6. CV Jakowatz, DE Wahl, PH Eichel, et al, Spotlight Mode Synthetic Aperture Radar: a Signal Processing Approach. (Kluwer Academic Publisher, Boston, 1996), pp. 252-267

7. I Cumming, F Wong, Digital Processing of Synthetic Aperture Radar Data: Algorithms and Implementation. (Norwood, Artech House, 2005)

8. WQ Wang, QC Peng, JY Cai, Waveform-diversity-based millimeter-wave UAV SAR remote sensing. IEEE Trans. Geosci. Remote Sensing. 47(3), 691-700 (2009)

9. RK Raney, H Runge, IG Cumming, et al, Precision of SAR processing using chirp scaling. IEEE Trans. Geosci. Remote Sensing. 32(4), 786-799 (1994)

10. GW Davidson, ID Cumming, MR Ito, A chirp scaling approach for processing squint model SAR data. IEEE Trans. Aerospace Electronic Syst. 32(1), 121-133 (1996)

11. MD Yang, DY Zhu, W Song, Comparison of two-step and one-step motion compensation algorithms for airborne synthetic aperture radar. Electron. Lett. 51(14), 1108-1110 (2015)

12. R Bamler, A comparison of range-doppler and wavenumber domain $S A R$ focusing algorithm. IEEE Trans. Geosci. Remote Sensing. 30(4), 706-713 (1992)

13. A Reigber, E Alivizatos, A Potsis, et al, Extended wavenumber-domain synthetic aperture radar focusing with integrated motion compensation. IET Radar Sonar Navigation. 153(3), 301-310 (2006)

14. KAC Macedo, R Scheiber, Precise topography- and aperture-dependent motion compensation for airborne SAR. IEEE Geosci. Remote Sensing Lett. 2(2), 172-176 (2005)

15. P Prats, KAC Macedo, A Reigber, et al, Comparison of topography- and aperture-dependent motion compensation algorithms for airborne SAR. IEEE Geosci. Remote Sensing Lett. 4(3), 349-353 (2007)

16. P Prats, A Reigber, JJ Mallorqui, Topography-dependent motion compensation for repeat-pass interferometric SAR systems. IEEE Geosci. Remote Sensing Lett. 2(2), 206-210 (2005)

17. S Perna, V Zamparelli, A Pauciullo, et al, Azimuth-to-frequency mapping in airborne SAR data corrupted by uncompensated motion errors. IEEE Geosci. Remote Sensing Lett. 10(6), 1493-1497 (2013)

18. A Potsis, A Reigber, J Mittermayer, et al, Sub-aperture algorithm for motion compensation improvement in wide-beam SAR data processing. Electron. Lett. 37(23), 1405-1407 (2001)

19. G Fornaro, E Sansosti, R Lanari, et al, Role of processing geometry in SAR raw data focusing. IEEE Trans. Aerospace Electron. Syst. 38(2), 441-454 (2002) 